\title{
Which Benefits and Harms of Using Fenugreek as a Galactogogue Need to Be Discussed during Clinical Consultations? A Delphi Study among Breastfeeding Women, Gynecologists, Pediatricians, Family Physicians, Lactation Consultants, and Pharmacists
}

\author{
Ramzi Shawahna $\mathbb{D D}^{1,2}$ Sara Qiblawi, ${ }^{3}$ and Haifa Ghanayem ${ }^{3}$ \\ ${ }^{1}$ Department of Physiology, Pharmacology and Toxicology, Faculty of Medicine and Health Sciences, An-Najah National University, \\ Nablus, State of Palestine \\ ${ }^{2}$ An-Najah BioSciences Unit, Centre for Poisons Control, Chemical and Biological Analyses, An-Najah National University, \\ Nablus, State of Palestine \\ ${ }^{3}$ Department of Medicine, Faculty of Medicine and Health Sciences, An-Najah National University, Nablus, State of Palestine
}

Correspondence should be addressed to Ramzi Shawahna; ramzi_shawahna@hotmail.com

Received 16 January 2018; Accepted 27 March 2018; Published 23 April 2018

Academic Editor: Sérgio Faloni De Andrade

Copyright (C) 2018 Ramzi Shawahna et al. This is an open access article distributed under the Creative Commons Attribution License, which permits unrestricted use, distribution, and reproduction in any medium, provided the original work is properly cited.

\begin{abstract}
Background. Breastfeeding women with hypogalactia are commonly recommended to use fenugreek as a galactogogue. This study aimed to achieve formal consensus among breastfeeding women and healthcare providers on which potential harms and benefits of using fenugreek need to be communicated and discussed during clinical consultations. Methods. A two-iterative round Delphi technique was used in two separate panels of breastfeeding women $(n=65)$ and healthcare providers $(n=56)$ to achieve formal consensus on a list of 24 and 16 items related to potential harms and benefits of fenugreek. Results. About $70 \%$ of the healthcare providers recommended quite often herbal remedies for breastfeeding women and about $68 \%$ of the women had been recommended to use herbal remedies many times by their healthcare providers. Consensus was achieved on 21 potential harms and 14 potential benefits of using fenugreek to enhance human milk supply that need to be discussed with breastfeeding women during consultations. Conclusion. Probably, potential harms and benefits of recommending fenugreek as herbal galactogogue for breastfeeding women seeking recommendations to increase their human milk supply need to be discussed during clinical consultations. Further observational studies are needed to assess what is being discussed in daily consultations when herbal remedies are recommended.
\end{abstract}

\section{Introduction}

Human milk has been recognized as the ideal form of enteral nutrition for term and preterm infants $[1,2]$. Exclusive breastfeeding for the first six months of life has been shown to confer substantial benefits to both the mother and her infant [2]. Therefore, global health authorities recommend exclusive breastfeeding for all infants in the first six months of life which might then be continued alongside other solid foods as long as the mother and her infant desire [3]. According to recent estimates, only $37 \%$ of infants younger than six months of age are nourished exclusively on human milk in low and middle income countries [2]. In the US and Australia, about half of the infants were receiving human milk at all by 6 months and in the UK, only one-third were doing so [2]. These low figures cannot be explained merely by weak intentions to breastfeed because in the UK, more than $80 \%$ of women expressed their intention to breastfeed their infants $[3,4]$. Certainly, some figures might be explained by insufficient human milk supply. 
Insufficient breast milk supply was frequently reported as the main reason for discontinuing breastfeeding $[5,6]$. Many women, particularly those who delivered preterm infants, suffer difficulties producing enough quantities of human milk. It is noteworthy mentioning here that even mothers of term infants under certain circumstances like maternal illness, cesarean delivery, excessive smoking, breast surgery, separation between mother and her infant, and psychosomatic illnesses might suffer insufficient human milk supply [3].

Nonpharmacological interventions remain the first line in managing insufficient human milk supply, although prescribing medications and recommending herbal galactogogues are common [7]. Women who discontinue breastfeeding as a result of insufficient human milk supply might be provided with adequate educational interventions regarding breastfeeding practices and/or might then be prescribed pharmacological agents to increase their human milk supply. Agents used to increase human milk supply are called galactogogues [5]. Metoclopramide and domperidone are the most commonly prescribed pharmacological galactogogues $[5,8,9]$. However, these agents have not received approval as safe and effective galactogogues from any health regulatory authority and currently are being used "off-label" $[10,11]$. In addition, these agents are excreted in human milk and thus bear potential side effects and harms to infants [1012]. Moreover, little guidance is available on the appropriate dosage of these agents when used as galactogogues [9, 13, 14]. Therefore, interventions to increase human milk supply using pharmacological agents might be complicated by safety concerns to women and their infants. Traditionally, herbal remedies have been viewed as good alternatives to prescription medications $[15,16]$.

In classical views, herbal remedies have been regarded as safe. Probably, this belief has emerged by advertising herbal remedies as mild, gentle, safe, and having unique attributes that are not found in prescription medications [15]. This myth was perpetuated by some healthcare providers when labeling herbal remedies as "natural" which are in turn mistakenly regarded as safe or in the worst case scenario, safer than prescription medications [17-19]. The myth that herbal remedies can never be harmful is perpetuated and commonly believed by many patients. However, this myth lacks scientific evidence. Herbal remedies contain chemicals that could resemble some active ingredients present in many prescription medications; thus, these chemicals would act by similar pharmacological mechanisms of action and have the ability to cause side effects and harm $[15,20]$. It is noteworthy mentioning that herbal remedies are like prescription medications, have intended indications, are contraindicated in some cases, should be used with caution in some patients, and are associated with side effects $[17,18]$. Therefore, herbal remedies should be recommended considering the 5 rights (right person, time, dose, frequency, and route of administration).

Herbal galactogogues have received considerable attention across different societies and cultures. Anecdotal reports of many herbal remedies supported their potential to enhance human milk supply. These herbal remedies include fenugreek, anise, caraway, fennel, milk thistle, and many others [16, 18, 21]. Fenugreek (Trigonella arabica Delile) which belongs to the pea family (Leguminosae) is the most widely used herbal galactogogue to enhance human milk supply in many countries [22]. Seeds of fenugreek, which is an annual herbaceous plant, are traditionally used as condiment and in folk medicine in many countries including the Indian subcontinent, China, and the Middle East [22]. A recent study in Kuwait showed that fenugreek was recommended for breastfeeding women with insufficient breast milk supply [23]. Anecdotal reports of the successful use of fenugreek as an herbal galactogogue have surfaced in 1940s. Little is known of the mechanism of action explaining how fenugreek might enhance milk supply. A theory suggested that fenugreek stimulate sweat production, and as the breast is a modified form of sweat gland, fenugreek might be able to stimulate the breast to supply an increasing amount of milk [21, 24]. There have been anecdotal reports of fenugreek increasing human milk supply in some 1200 breastfeeding women within 24-72 hours after consumption [24, 25]. Once the breast is stimulated, fenugreek consumption can be stopped as far the breast is stimulated and emptying continued. Fenugreek as galactogogue might be consumed in 2-3 capsules 3 times daily and each capsule might contain a variable quantity of fenugreek. At present, requirements for herbal products have not been standardized for consumption by patient [24]. It is noteworthy mentioning that the use of fenugreek is not free from side effects and has been associated with health related effects like excessive sweating, diarrhea, and worsening of asthma symptoms.

In modern healthcare delivery, patients are informed about the potential harms and benefits of therapeutic alternatives in order to develop their preferences. In general, making a decision on therapeutic alternatives involves balancing their potential benefits against their potential harms, taking into account the preferences of the patients. The benefits of informing patients are multifold, including better experienced quality of life, coping with side effects, and prevention of overestimation of the impact of therapy on cure [15]. Therefore, healthcare providers like gynecologists/obstetricians, pediatricians, lactation consultants, family physicians, and pharmacists who are often consulted by breastfeeding women seeking recommendations to enhance their human milk supply should discuss herbal galactogogues balancing their potential benefits again potential harms in case they wanted to opt for herbal remedies considering the preferences of the women concerned. Little was narrated on the potential harms and benefits of using fenugreek to enhance human milk supply in breastfeeding women that should be discussed during clinical consultations from the viewpoints of breastfeeding women, gynecologists/obstetricians, pediatricians, family physicians, lactation consultants, and pharmacists who are often consulted by breastfeeding women seeking recommendations to enhance their human milk supply. In general, recommendations on which potential harms and benefits of using fenugreek to communicate to and discuss with patients during clinical consultations are lacking. The aim of this study was to fill this gap in the literature. 
The aims of this study was to achieve consensus among breastfeeding women, gynecologists/obstetricians, pediatricians, family physicians, lactation consultants, and pharmacists who are often consulted by breastfeeding women seeking recommendations to enhance their human milk supply on which potential harms and benefits of using fenugreek as a galactogogue that need to be communicated to and discussed with breastfeeding women during clinical consultations in which a decision to use fenugreek would be taken. This consensual core list of potential harms and benefits might promote congruence in daily healthcare delivery.

\section{Materials and Methods}

2.1. Gathering Information on Herbal Galactogogues Recommended in Clinical Practice. We contacted and interviewed 10 key contact healthcare providers who were often consulted by breastfeeding women seeking recommendations to use herbal galactogogues to enhance their human milk supply. We also interviewed 5 women who previously have sought recommendations and used herbal galactogogues to enhance their human milk supply.

The key contact healthcare providers were asked to provide their consent to include their initials and details as experts who were interviewed in this study. Participants were given the option to remain anonymous upon their desire. Key contacts provided their age, gender, academic degrees, specialty, number of years in practice, approximate number of breastfeeding women cared for on a monthly basis, herbal galactogogues they often recommend, the potential harms, and benefits of herbal galactogogues that need to be communicated to and discussed with breastfeeding women during the clinical consultations.

The key contact women were asked to provide their consent to include their initials and details as experts who were interviewed in this study. Women were also given the option to remain anonymous upon their desire. Women were asked to provide their age, academic degrees, employment status, and the potential harms and benefits of galactogogues that need to be communicated to and discussed with breastfeeding women during the clinical consultations. The detailed sociodemographic and practice details of the interviewees are provided as Supplementary Materials (Table S1).

Healthcare providers and women narrated their experience with herbal galactogogues in terms of benefits and harms. Herbal galactogogues mentioned by the interviewees are listed in Supplementary Materials (Table S2). All interviewees (healthcare providers and women) mentioned fenugreek as one of the most frequently recommended herbal galactogogues. As all interviewees mentioned fenugreek as a galactogogue, we decided to gather all potential harms and benefits of this herbal galactogogue that need to be communicated to and discussed with breastfeeding women during the clinical consultations between breastfeeding women and their caring healthcare providers in which fenugreek is to be recommended. All potential harms and benefits mentioned by the interviewees were collected. An extensive literature review was then conducted to gather other potential harms and benefits of using fenugreek that could be found in other studies $[4,6,12,13,17,18,21,22,24-46]$. All potential harms and benefits found in the previous studies were noted. Potential harms and benefits collected were rephrased into statements. We discarded all potential harms and benefits related to costs, convenience, and inconvenience. Statements were piloted for clarity and comprehensibility with 5 medical students and 5 lay persons. Some statements were revised based on the feedback of the pilot and all statements were compiled into a questionnaire.

2.2. The Consensual Technique. In this study, we used the Delphi technique as a tool to achieve formal consensus among panelists on which potential harms and benefits of using fenugreek by breastfeeding women to enhance their human milk supply should be communicated to and discussed with breastfeeding women during the clinical consultations between breastfeeding women and their caring healthcare providers. Recently, this formal consensus technique has evolved as one of the most frequently employed techniques in achieving consensus on issues lacking consensus in healthcare [15, 47-49]. This technique has many advantages over other techniques like round table meeting, focus, and nominal groups. The advantages of this technique include guarding the anonymity of the participants, ability to recruit panelists from different locations, convenience, saving the costs of bringing the panelists to a round table meeting, and immunity against individual domination of the discussion and influencing opinions of other panelists. The Delphi technique combines both quantitative as well as qualitative methods in which a multiround questionnaire system is completed in two or more iterative stages, known as rounds, over a period of time within one or more panels until consensus is achieved [50]. The panelists are often requested to express the level of their disagreement or agreement with a list containing items in a questionnaire. Consensus is defined a priori and items on which consensus was not reached in one round are included in a revised questionnaire for a subsequent round and the process is continued until reaching a conclusion that consensus on the remaining items is no longer likely to be achieved [15, $47-$ 49]. Sharing statistical summaries and comments with the panelists in a trial to decrease the number of rounds needed to reach consensus on the items included is commonly practiced.

As the views and opinions of women and healthcare providers could be different from each other, we sought consensus in two separate panels [15]. A panel included healthcare providers who are often consulted by breastfeeding women seeking recommendations to increase their human milk supply and the other panel was composed of women who sought recommendations and used herbal galactogogues to enhance their human milk supply.

2.3. Panel of Healthcare Providers. A judgmental sampling technique was used to recruit panelists who were healthcare providers that were often consulted by breastfeeding women seeking recommendations to increase their human milk supply. Potential panelists were identified by personal contacts in the field. As breastfeeding women seeking recommendations 
to increase their human milk supply often consult gynecologists/obstetricians, lactation consultant nurses, pediatricians, family medicine specialists, and pharmacists, we aimed to recruit panelists with these specialties. Because the Delphi technique implies that the panelists have to be rich with experience and information to narrate, it is well-established that selection and recruitment of the panel members are among the most captious steps in the Delphi technique [15]. In the current study, panelists were approached and invited to participate as panel members based on their qualifications, specialty, and experience in the field of recommending herbal galactogogues for breastfeeding women seeking recommendations to enhance their human milk supply. Field researchers approached in person and invited the potential panelists to participate as panel members in the current study. Field researchers explained the design and objectives of the study to potential panelists and obtained their verbal consent before participation. The inclusion criteria were (1) having a basic or advanced qualification in a healthcare specialty related to being consulted by breastfeeding women seeking recommendations to enhance their human milk supply, (2) having a license to practice in Palestine, (3) having 5 or more years of practicing experience in a healthcare establishment attended by breastfeeding women seeking recommendations to enhance their human milk supply, and this was important as possessing previous knowledge of the subject being researched is a critical prerequisite for a panelist to take part in the Delphi technique [15], (4) consultation with 5 or more breastfeeding women on a monthly basis, (5) knowledge of the use of herbal galactogogues in enhancing human milk supply. In this study, 56 panelists were recruited and participated in the panel of healthcare providers. The panelists were not offered any financial incentives.

2.4. Panel of Women. In this study, snowball sampling was used to identify and recruit women who sought recommendations and used herbal galactogogues to enhance their milk supply. Potential panelists were identified using personal contacts in the field. Potential panelists were approached by field researchers in person and invited them to participate in this study. The field researchers explained the design and objectives of the study to the potential panelists and obtained their verbal consent before they were recruited to the panel. Women were invited and recruited to the panel when they met the inclusion criteria of (1) having breastfed at least one infant, (2) having been recommended at least once to use herbal galactogogues to enhance their human milk supply, (3) using one or more herbal galactogogue to enhance human milk production, and (4) willingness to take part in the current study. In this study, 65 women were recruited to the panel. Again, participants were not offered any financial incentives.

\subsection{The Iterative Delphi Technique Rounds}

2.5.1. Delphi Round 01. In the first Delphi round, the questionnaire was given by hand to all 56 healthcare providers and 65 women. The questionnaire consisted of 2 sections. In the 1st section, the panelists were requested to disclose their sociodemographic details. The healthcare professionals provided their gender, age, academic qualifications, number of years in practice, specialty, how often they recommended herbal galactogogues for breastfeeding women in their clinical practice, and how often they communicated and discussed harms and benefits of herbal galactogogues that breastfeeding women might be consuming during clinical consultations. Female healthcare professionals were also requested to provide if they have breastfed before, and the number of infants they breastfed. Women were requested to provide their age, educational level, employment status, number of infants they breastfed, how often they have been recommended by their healthcare providers to use herbal remedies to enhance their human milk supply, and if they liked to have enough discussion with their healthcare providers on the potential harms and benefits of using herbal remedies during breastfeeding. The 2nd section of the questionnaire contained a list of 24 and 16 items related to potential harms and benefits, respectively, of using fenugreek as a herbal galactogogue to enhance human milk supply and the panelists were requested to express the degree to which they disagree or agree that each presented item needs to be communicated to and discussed with breastfeeding women during consultations on a Likert scale of 9 points $[15,47-49]$. When the panelists scored $1-3$, this indicated that they disagree with the importance of communicating and discussing the presented potential harm or benefit during the clinical consultation; that is, they are of the opinion that the presented potential harm or benefit should not be communicated to and discussed with breastfeeding women during the consultations. When the panelists scored 7-9, this indicated that they agree with the importance of communicating and discussing the presented potential harm or benefit to breastfeeding women during the clinical consultation; that is, they are of the opinion that the proposed potential harm or benefit should be communicated to and discussed with breastfeeding women during the consultation. When the panelists scored 4-6, this indicated that the panelists partially agreed with the importance of communicating and discussing the presented potential harm or benefit during the clinical consultation; that is, the panelists are inconclusive either the presented potential harm or benefit should be communicated to and discussed with breastfeeding women or not during the consultations. In this study, the panel members were requested and encouraged to add written comments to justify and/or qualify their scores on the Likert scale as in previous studies [15, 47-49].

\subsubsection{Definition of Consensus and Analysis of the Scores.} Scores were analyzed using an Excel Sheet (Microsoft Excel 2013). The first quartile (Q1), median (Q2), third quartile (Q3), and the interquartile range (IQR) were computed for each item. Scores of both panels were analyzed separately. The data were analyzed using the same definitions of consensus used in previous studies [15, 47-49]. Briefly, the item included the list of important harms or benefits that need to be communicated to and discussed with breastfeeding women during the consultation when the median score fell between 7 and 9 and the interquartile range (IQR) fell between 1 and 2 and the item was excluded from the list of important harms 
or benefits that need to be communicated to and discussed with breastfeeding women during the consultation when the median score fell between 1 and 3 and the IQR fell between 1 and 2. However, the item was considered equivocal when the median score fell between 4 and 6 or the IQR was larger than 2. Equivocal items were included in a revised questionnaire for a subsequent Delphi round. In this study, consensus was based on at least $80 \%$ of the scores of the panelists in each panel separately.

2.5.3. Delphi Round 02. A revised questionnaire containing all equivocal items was subjected to a second Delphi round. In a trial to reduce the number of Delphi rounds needed to reach consensus, we provided the panelists with (1) the median score and the IQR for each potential harm or benefit, (2) reminder of their own scores in the previous Delphi round, and (3) summary of the comments made by the panelists either to justify or qualify their scores.

Scores in this round were computed and analyzed according to the same definitions used in the previous Delphi round. After analyzing the scores and comments obtained in the second Delphi round, we came to a conclusion that it was unlikely that consensus would be achieved if we would conduct further Delphi rounds.

2.6. Ethical Considerations. This study received ethical approval from the Institutional Review Board (IRB) committee of An-Najah National University. We obtained verbal consent from all panelists before they participated in the current study. All views, opinions, and scores of the panelists weighed equally in the analysis.

\section{Results}

3.1. Response Rate. Questionnaires were completed by 56 healthcare providers who are often consulted by breastfeeding women and 65 women who breastfed before in the first Delphi round; therefore, the response rate was $100 \%$. However, in the second Delphi round, $48(85.7 \%)$ of the healthcare providers and $40(61.5 \%)$ of the women completed and returned the questionnaire.

\subsection{Characteristics of the Panelists Who Took Part in the Study}

3.2.1. The Panel of Healthcare Providers. In this study, the panelists who were healthcare providers were of different age groups, belonged to both genders, had variable number of years in practice, had different academic qualifications, and had various specialties. More than half of the panelists were male in gender, physicians, and 40 years, and older. About $56 \%$ of the panelists were either gynecologists/obstetricians, pediatricians, or family medicine specialists. About $59 \%$ of the panelists were in practice for 10 or more years. The detailed characteristics of the panelists are shown in Table 1 .

3.2.2. The Panel of Women. The women who took part as panelists in this study were of different age groups and had different educational levels and employment status. The majority of the women (about $85 \%$ ) had a university degree
TABLE 1: Sociodemographic and practice details of the healthcare providers who are often consulted by breastfeeding women $(n=56)$.

\begin{tabular}{|c|c|c|}
\hline Variable & $n$ & $\%$ \\
\hline \multicolumn{3}{|l|}{ Gender } \\
\hline Male & 30 & 53.6 \\
\hline Female & 26 & 46.4 \\
\hline \multicolumn{3}{|l|}{ Age (years) } \\
\hline$<40$ & 30 & 53.6 \\
\hline$\geq 40$ & 26 & 46.4 \\
\hline \multicolumn{3}{|l|}{ Have you breastfed before ${ }^{a}$} \\
\hline Yes & 19 & $73.1^{\mathrm{b}}$ \\
\hline No & 7 & $26.9^{\mathrm{b}}$ \\
\hline \multicolumn{3}{|l|}{ Number of infants breastfed ${ }^{\mathrm{a}}$} \\
\hline 0 & 7 & $26.9^{b}$ \\
\hline 1 & 4 & $15.4^{\mathrm{b}}$ \\
\hline 2 & 3 & $11.5^{\mathrm{b}}$ \\
\hline$\geq 3$ & 12 & $46.2^{\mathrm{b}}$ \\
\hline \multicolumn{3}{|l|}{ Academic qualifications } \\
\hline B.S. & 21 & 37.5 \\
\hline M.S. & 5 & 8.9 \\
\hline M.D. & 28 & 50.0 \\
\hline Ph.D. & 2 & 3.6 \\
\hline \multicolumn{3}{|l|}{ Specialty } \\
\hline Gynecology/obstetrics & 10 & 17.9 \\
\hline Pediatrics & 5 & 8.9 \\
\hline Family medicine & 16 & 28.6 \\
\hline Lactation consultant nurse & 13 & 23.2 \\
\hline Pharmacist & 12 & 21.4 \\
\hline \multicolumn{3}{|l|}{ Number of years in practice } \\
\hline $5-9$ & 23 & 41.1 \\
\hline$\geq 10$ & 33 & 58.9 \\
\hline \multicolumn{3}{|c|}{$\begin{array}{l}\text { How often do you recommend herbal } \\
\text { galactagogues for breastfeeding women? }\end{array}$} \\
\hline Quite often & 39 & 69.6 \\
\hline Sometimes & 17 & 30.4 \\
\hline \multicolumn{3}{|c|}{$\begin{array}{l}\text { How often do you discuss herbal galactagogues } \\
\text { that breastfeeding women could be using during } \\
\text { your consultations with them? }\end{array}$} \\
\hline Quite often & 32 & 57.1 \\
\hline Sometimes & 24 & 42.9 \\
\hline
\end{tabular}

${ }^{\mathrm{a}}$ The question was for healthcare providers who were female in gender.

${ }^{\mathrm{b}}$ Percentages were based on the number of female panelists; B.S.: Bachelor of Science, M.S.: Master of Science, M.D.: Doctor of Medicine, and Ph.D.: Doctor of Philosophy.

and were 25 years and older. About $43 \%$ of the women breastfed 3 or more infants. The detailed variables of the women panelists who participated in this study are shown in Table 2 .

3.3. Use of Fenugreek for Enhancing Human Milk Supply. About $70 \%$ of the healthcare provider panelists stated that 
TABLE 2: Sociodemographic details of the women who participated in this study $(n=65)$.

\begin{tabular}{|c|c|c|}
\hline Variable & $n$ & $\%$ \\
\hline \multicolumn{3}{|l|}{ Age (years) } \\
\hline$<25$ & 10 & 15.4 \\
\hline$\geq 25$ & 55 & 84.6 \\
\hline \multicolumn{3}{|l|}{ Educational level } \\
\hline School & 16 & 24.6 \\
\hline Bachelor's degree & 37 & 56.9 \\
\hline Master's degree & 12 & 18.5 \\
\hline \multicolumn{3}{|l|}{ Employment status } \\
\hline Employed & 39 & 60.0 \\
\hline Unemployed & 26 & 40.0 \\
\hline \multicolumn{3}{|c|}{ Number of infants breastfed } \\
\hline 1 & 22 & 33.8 \\
\hline 2 & 15 & 23.1 \\
\hline$\geq 3$ & 28 & 43.1 \\
\hline \multicolumn{3}{|c|}{$\begin{array}{l}\text { How often have you been recommended by your } \\
\text { healthcare provider to use herbal remedies for } \\
\text { enhancing your human milk supply? }\end{array}$} \\
\hline Many times & 44 & 67.7 \\
\hline Once or a few times & 21 & 32.3 \\
\hline \multicolumn{3}{|c|}{$\begin{array}{l}\text { Do you like to have enough discussion with your } \\
\text { healthcare provider on the potential harms and } \\
\text { benefits of using herbal remedies? }\end{array}$} \\
\hline Always & 43 & 66.2 \\
\hline Sometimes & 22 & 33.8 \\
\hline
\end{tabular}

they recommended quite often herbal remedies for breastfeeding women. About $68 \%$ of the women had been recommended many times by their healthcare providers to use herbal remedies for enhancing their human milk supply.

About $57 \%$ of the panelists discussed quite often herbal remedies that breastfeeding women could be using during their consultations with them. About $66 \%$ of the women stated that they would always like to have enough discussion with their healthcare providers on the potential harms and benefits of using herbal remedies for enhancing their human milk supply.

3.4. Potential Harms of Using Fenugreek to Enhance Human Milk Supply That Need to Be Communicated to and Discussed with Breastfeeding Women during the Clinical Consultation. In this study, consensus was achieved in both panels on 21 potential harms of using fenugreek to enhance human milk supply that need to be communicated to and discussed with breastfeeding women during the consultation. The detailed list of these items is shown in Table 3.

In general, there was consensus on 6 potential harms related to the anticoagulant effects of fenugreek, 3 potential harms related to the increased risk of abortion associated with using fenugreek, 4 potential harms related to comorbidities, 3 potential harms related to the effects of fenugreek on the blood pressure, 2 potential harms related to the effects of fenugreek on the blood glucose level, and 3 other potential harms related to the side effects of fenugreek.

3.5. Potential Benefits of Using Fenugreek to Enhance Human Milk Supply That Need to Be Communicated to and Discussed with Breastfeeding Women during the Consultation. In this study, consensus was achieved in both panels on 14 potential benefits of using fenugreek to enhance human milk supply that need to be communicated to and discussed with breastfeeding women during the consultation. A detailed list of these potential benefits is shown in Table 4 .

In general, there was consensus on the potential benefits of fenugreek related to enhancing human milk supply and fertility. Consensus was also achieved to communicate and discuss other potential benefits of fenugreek related to its antioxidant, chemoprotective, immunomodulatory, antidepressant, and anti-infective properties with breastfeeding women.

3.6. Potential Harms and Benefits of Using Fenugreek to Enhance Human Milk Supply That Need or Need Not to Be Communicated to and Discussed with Breastfeeding Women during the Consultation Depending on the Individual Clinical Situation's Need. Consensus was not achieved on 3 potential harms and 2 potential benefits of using fenugreek to enhance human milk supply. These equivocal items are listed in Table 5. Whether to communicate and discuss these items during a clinical consultation was left to the choice of the healthcare provider and depending on the individual's needs.

\section{Discussion}

In the present study, we developed a consensual core list of important potential harms and benefits of using fenugreek as herbal galactogogue that should be communicated to and discussed with breastfeeding women seeking recommendations to increase their human milk supply from their caring healthcare providers in daily practice in two separate panels of women and healthcare providers. To the best of our knowledge, this consensual core list is the first attempt to develop guidance for healthcare providers to consult when recommending fenugreek-based herbal remedies to promote human milk supply in breastfeeding women seeking recommendations to enhance their human milk supply.

When gold standards are not existent, consensual techniques might provide alternative methods to reduce bias, enhance transparency, and validity of judgmental methods when developing certain criteria [15]. We believe that this consensual core list should appeal to healthcare providers and might be consulted to guide communicating and discussing potential harms and benefits of using fenugreek to promote human milk supply in breastfeeding women seeking recommendations to enhance their milk supply. Judgmental sampling was used to recruit panelists for the panel of healthcare providers and snowball sampling was used to recruit panelists for the panel of women. These nonprobability sampling techniques have long been regarded as biased [51]. However, for this study design and objectives, probability randomized 
TABLE 3: Potential harms of using fenugreek to enhance human milk supply that need to be communicated to and discussed with breastfeeding women during the clinical consultation.

\begin{tabular}{llr}
\hline $\begin{array}{l}\text { Item } \\
\#\end{array}$ & Potential harms & Round on which consensus was achieved \\
& Panel of healthcare providers & Panel of women \\
\hline
\end{tabular}

Fenugreek has anticoagulant effects

1 Breastfeeding women who have a history of any clotting related disorder need to be warned not to take fenugreek

2 Breastfeeding women who have a history of vaginal bleeding disorder need to be warned not to take fenugreek

3

Breastfeeding women who are at risk of any bleeding disorder need to be warned not to take fenugreek

4 Breastfeeding women need to be warned that fenugreek might be associated with menstrual breakthrough bleeding

5 Breastfeeding women who are on anticoagulants need to be warned not to take fenugreek

6 Breastfeeding women who are on non-steroidal anti-inflammatory drugs

(NSAIDs) need to be warned not to take fenugreek

Panel of healthcare providers Panel of women

\section{Fenugreek might be associated with abortion}

$7 \quad$ Women planning to become pregnant need to be warned that fenugreek is a potential utero-stimulant and might cause spontaneous abortion

Women with a history of previous miscarriage need to be warned not to take fenugreek

9 Women planning to become pregnant need to be warned that fenugreek might impair fetal development

Risks associated with using fenugreek on other co-morbidities

Breastfeeding women need to be warned that fenugreek might cause nausea and vomiting

Breastfeeding women need to be warned that fenugreek might cause diarrhea in the mother and her breastfed infant

12 Breastfeeding women with a history of asthma need to be warned that fenugreek might worsen the symptoms of their asthma

13 Breastfeeding women need to be warned that fenugreek might cause dehydration

Fenugreek could be associated with hypotension

14

Breastfeeding women with a history of or at risk of hypotension need to be warned not to take fenugreek

Breastfeeding women with a history of or at risk of dizziness need to be warned not to take fenugreek

Breastfeeding women who are on anti-hypertensive medications need to be warned not to take fenugreek

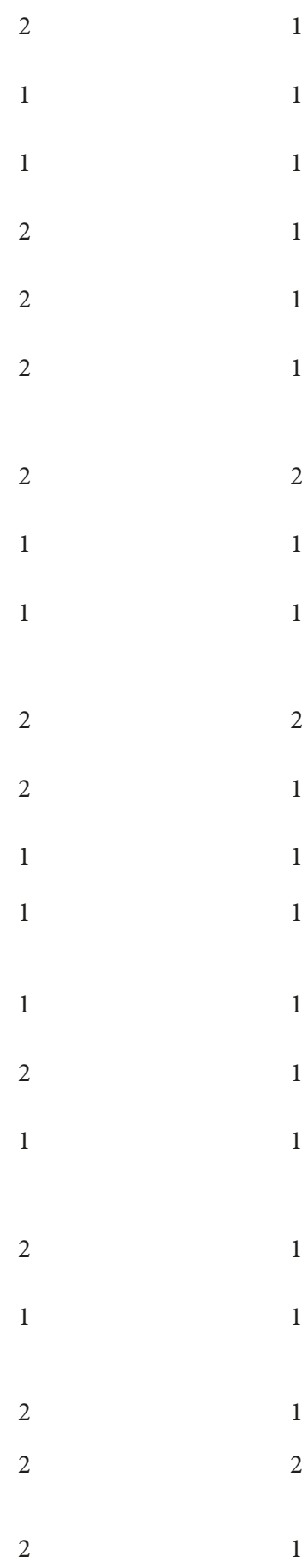

Fenugreek could be associated with hypoglycemia

17 Breastfeeding women with a history of or at risk of hypoglycemia need to be warned not to take fenugreek

18 Diabetic breastfeeding women whose disease is controlled by medications or insulin need to be warned not to take fenugreek

Other adverse effects

19 Breastfeeding women need to be warned that fenugreek might cause fever

20 Breastfeeding women need to be warned that fenugreek might cause excessive sweating

Breastfeeding women taking diuretics, laxatives, mineralocorticoids, and/or

21 other hypokalemic agents need to be warned that fenugreek may worsen hypokalemia

1

1

1

1

1

1

2

1

1

2

1

1

1

1

1

1

1

1

1

2

1 
TABLE 4: Potential benefits of using fenugreek to enhance human milk supply that need to be communicated to and discussed with breastfeeding women during the clinical consultation.

\begin{tabular}{|c|c|c|c|}
\hline \multirow{2}{*}{$\begin{array}{l}\text { Item } \\
\#\end{array}$} & \multirow{2}{*}{ Potential benefits } & \multicolumn{2}{|c|}{ Round on which consensus was achieved } \\
\hline & & Panel of healthcare providers & Panel of women \\
\hline 1 & $\begin{array}{l}\text { Breastfeeding women might be informed that fenugreek can be beneficial in } \\
\text { enhancing their human milk production }\end{array}$ & 1 & 1 \\
\hline 2 & $\begin{array}{l}\text { Breastfeeding women might be informed that fenugreek might improve their } \\
\text { fertility }\end{array}$ & 2 & 2 \\
\hline 3 & $\begin{array}{l}\text { Breastfeeding women might be informed that fenugreek has antioxidant } \\
\text { properties }\end{array}$ & 2 & 2 \\
\hline 4 & Breastfeeding women might be informed that fenugreek has estrogenic effects & 2 & 1 \\
\hline 5 & $\begin{array}{l}\text { Breastfeeding women might be informed that fenugreek has immunomodulatory } \\
\text { effect }\end{array}$ & 1 & 1 \\
\hline 6 & $\begin{array}{l}\text { Breastfeeding women might be informed that fenugreek has chemo-protective } \\
\text { effect against breast cancer }\end{array}$ & 1 & 1 \\
\hline 7 & $\begin{array}{l}\text { Breastfeeding women might be informed that fenugreek may decrease plasma } \\
\text { cholesterol and triglycerides levels }\end{array}$ & 1 & 1 \\
\hline 8 & $\begin{array}{l}\text { Breastfeeding women might be informed that fenugreek may have antidepressant } \\
\text { activity }\end{array}$ & 2 & 1 \\
\hline 9 & $\begin{array}{l}\text { Breastfeeding women might be informed that fenugreek may have antibacterial } \\
\text { activity }\end{array}$ & 1 & 1 \\
\hline 10 & $\begin{array}{l}\text { Breastfeeding women might be informed that fenugreek may have antifungal } \\
\text { activity }\end{array}$ & 1 & 1 \\
\hline 11 & $\begin{array}{l}\text { Breastfeeding women might be informed that fenugreek could decrease their } \\
\text { appetite, especially those with a history of eating disorders }\end{array}$ & 2 & 1 \\
\hline 12 & Breastfeeding women might be informed that fenugreek can enhance weight loss & 2 & 1 \\
\hline 13 & $\begin{array}{l}\text { Breastfeeding women might be informed that fenugreek might have antipyretic } \\
\text { activity }\end{array}$ & 2 & 1 \\
\hline 14 & $\begin{array}{l}\text { Breastfeeding women might be informed that fenugreek may alleviate symptoms } \\
\text { of ulcer }\end{array}$ & 2 & 1 \\
\hline
\end{tabular}

TABLE 5: Potential harms and benefits of using fenugreek to enhance human milk supply that need or need not to be communicated to and discussed with breastfeeding women during the consultation depending on the individual clinical situation's need.

\begin{tabular}{|c|c|c|c|c|c|c|c|c|c|}
\hline \multirow{3}{*}{ Item \# } & & \multicolumn{4}{|c|}{$\begin{array}{l}\text { Panel of healthcare } \\
\text { providers }\end{array}$} & \multicolumn{4}{|c|}{ Panel of women } \\
\hline & & \multicolumn{2}{|c|}{ Round 1} & \multicolumn{2}{|c|}{ Round 2} & \multicolumn{2}{|c|}{ Round 1} & \multicolumn{2}{|c|}{ Round 2} \\
\hline & & M & IQR & M & IQR & M & IQR & M & IQR \\
\hline & Potential harms & & & & & & & & \\
\hline 1 & $\begin{array}{l}\text { Breastfeeding women need to be warned that } \\
\text { fenugreek may induce thirst }\end{array}$ & 5 & 2 & 5 & 3 & 6 & 2 & 5 & 3 \\
\hline 2 & $\begin{array}{l}\text { Breastfeeding women need to be warned that } \\
\text { fenugreek may be associated with maple syrup } \\
\text { like urine }\end{array}$ & 4 & 3 & 5 & 2 & 5 & 2 & 6 & 3 \\
\hline 3 & $\begin{array}{l}\text { Breastfeeding women need to be warned that } \\
\text { fenugreek may be associated with maple syrup } \\
\text { like sweat }\end{array}$ & 5 & 2 & 4 & 3 & 4 & 3 & 4 & 2 \\
\hline & Potential benefits & & & & & & & & \\
\hline 1 & $\begin{array}{l}\text { Breastfeeding women might be informed that } \\
\text { fenugreek may have antiparkinsonian activity }\end{array}$ & 4 & 4 & 5 & 3 & 6 & 2 & 6 & 3 \\
\hline 2 & $\begin{array}{l}\text { Breastfeeding women might be informed that } \\
\text { fenugreek may improve memory and cognition }\end{array}$ & 4 & 2 & 4 & 3 & 5 & 3 & 5 & 3 \\
\hline
\end{tabular}

M: median, IQR: interquartile range. 
sampling techniques were not feasible. Moreover, judgmental and snowball sampling techniques permitted the recruitment of panelists with prior knowledge of the subject being investigated who were rich in experience to narrate [15, 4749]. The panel of healthcare providers was composed of gynecologists/obstetricians, pediatricians, family physicians, lactation consultants, and pharmacists. Those healthcare professionals would normally be consulted by breastfeeding women seeking recommendations to increase their human milk supply $[17,52]$. Women who were recruited for the panel of women experienced inadequate human milk supply sought recommendations from healthcare providers and used herbal galactogogues.

The number of panelists in the panel of healthcare providers and panel of women was slightly larger than those used in previous studies in which consensus was sought on issues in healthcare [15, 47-49]. Currently, there is no consensus on the number of panelists in a panel of experts. Panel sizes varied greatly in previous studies and the sizes ranged from 10 over 1000 panel members [51].

In this study, a consensual core list of potential harms and benefits of using fenugreek as herbal galactogogue was developed to guide healthcare providers on what harms and benefits to discuss and/or address during the clinical consultation when opting to recommend fenugreek for breastfeeding women seeking recommendations to increase their human milk supply. Guidelines on what healthcare providers should communicate and discuss in terms of potential harms and benefits are currently lacking. We believe this consensual core list should help healthcare providers and change their behaviors during consultations with breastfeeding women seeking recommendations to increase their human milk supply. It has been argued that professionals would change behavior in response to recommendations they agree with rather than recommendations they do not agree with $[15,47-$ 49].

The use of herbal remedies was reported to be high among women in Palestine [31, 53]. In this study, about $68 \%$ of the women reported that they were recommended to use herbal galactogogues many times. Similarly, about $70 \%$ of the healthcare providers reported that they recommend quiet often herbal galactogogues for breastfeeding women seeking recommendations to increase their human milk supply. Our findings were consistent with those previously reported by Bazzano et al. in the US, in which $70 \%$ of the healthcare providers surveyed indicated that they often recommend galactogogues [52]. Similarly, fenugreek was the most frequently recommended herbal galactogogue in Bazzano's study. In this study, about $68 \%$ of the women reported that they always wanted to have enough discussion with their caring healthcare providers on the potential harms and benefits of herbal remedies. Findings of this study were consistent with those reported in a previous study in which $76 \%$ of pregnant women stated that they would like to have enough discussion on the benefits and harms of ginger when recommended to alleviate symptoms of nausea and vomiting of pregnancy [15]. In this study, inclusion women who experienced human milk insufficiency and used herbal galactogogues in the panel of women ensured inclusion of the insecurities and concerns breastfeeding women would like their caring healthcare providers to address during clinical consultations. Interestingly, about $57 \%$ of the healthcare providers reported that they quite often address potential harms and benefits of herbal remedies during consultations with breastfeeding women.

In this study, the response rate was high in both Delphi rounds. This was consistent with other studies seeking consensus on issues in healthcare using the Delphi technique [15, 47-49]. This strength adds to the validity of the findings reported in this study. The panel of healthcare providers included panelists of both genders, different age groups, geographical locations, practice settings, specialties, and number of years in practice (Table 1). The panel of women included panelists from different geographical locations, age groups, number of breastfed infants, educational levels, and employment status (Table 2). This diversity adds to the strength and validity of the findings reported in this study.

In this study, consensus was achieved on potential harms related to the anticoagulant potential of fenugreek that need to be discussed and/or addressed during the clinical consultation (Table 3). These findings were consistent with those reported in another study in which consensus was achieved among healthcare professionals on addressing the potential harms and benefits of using ginger to manage nausea and vomiting of pregnancy, especially harms related to the anticoagulant potential of ginger [15]. Not surprisingly, patients were previously reported to want to hear more from their healthcare providers on the best ways to make out of the therapies they are taking $[54,55]$. The anticoagulant effects of fenugreek were previously reported. A recent study showed that aqueous extract of fenugreek inhibited blood coagulation process in vitro and increased prothrombin time in a dose dependent manner in blood samples obtained from healthy individuals [41]. Drug-herb interaction between fenugreek and warfarin was also reported [26]. Professional groups like the American Society of Anesthesiologists have advised patients to stop consuming herbal therapies 2-3 weeks prior to surgery as a safety precaution to avoid risks of bleeding $[15,32]$. Findings of this study suggested that both healthcare providers and women wanted the risks of bleeding associated with the use of fenugreek by breastfeeding women to communicate and discuss during the consultation in which fenugreek is recommended to be used. Informed breastfeeding women could be in a better position to decide whether to use fenugreek or opt for another safer alternative.

In this study, the panelists were of the opinion that the risks of abortion associated with using fenugreek should be communicated to and discussed with breastfeeding women during the consultations. Again, these findings were consistent with those reported in a previous study in which pregnant women and gynecologists agreed that the risks of abortion associated with using ginger for nausea and vomiting of pregnancy should be addressed during clinical consultations [15]. Previous studies showed that aqueous extract of fenugreek had potential teratogenic effects in humans and animals $[33,39]$. Health regulatory bodies tend to recommend avoidance of herbal remedies even when the risks associated with their use are inconclusive. As a 
good example here, the German E Commission and the Finnish Food Safety Authority recommended that pregnant women should avoid ginger even though the risks of abortion associated with using ginger by pregnant women were largely inconclusive [56]. There could be cases in which breastfeeding women could become pregnant. The panelists in this study were of the opinion to warn women of these potential risks during the clinical consultations. Conservative views imply that women should be warned even when the potential risks are still inconclusive $[38,42]$.

The use of fenugreek could worsen the symptoms of some comorbidities. For example, fenugreek could worsen the symptoms of asthma [38, 42]. It has been recommended that individuals with chronic asthma and allergy should avoid consumption of fenugreek $[28,38]$. Therefore, in this study, the panelists were of the opinion that this risk should be communicated to and discussed with breastfeeding women during consultations. Many breastfeeding women could be asthmatics and should be warned of this potential harm of using fenugreek. Again, breastfeeding women should be warned that fenugreek could cause nausea and vomiting which could be disturbing to the breastfeeding women and could have negative effects on their reported quality of life [39]. Fenugreek could be associated with diarrhea and excessive sweating for the breastfeeding women and their breastfed infants [34]. Severe diarrhea and excessive sweating could result in huge fluid loss that might lead to dehydration as well as serious consequences on the health of infants. These risks should be communicated to and discussed with breastfeeding during the consultations.

The findings of this study suggested that the risks associated with the effects of fenugreek on the blood pressure, blood glucose, and potassium levels should be communicated to and discussed with breastfeeding women during the consultations $[29,30,37,40]$. Some breastfeeding women could be at risk of hypotension or hypoglycemia and should be warned against these risks when using fenugreek. The blood pressure and blood glucose levels of some breastfeeding women might be controlled by medications. Using fenugreek might have negative consequences of these controlled levels and hence, breastfeeding women at risk should be warned. Similarly, some breastfeeding women could be taking diuretics, laxatives, mineralocorticoids, or other hypokalemic agents. The panelists in this study were of the opinion that breastfeeding women should be warned that fenugreek might worsen their hypokalemia.

The panelists in this study agreed that benefits related to enhancing human milk supply should be communicated to and discussed with breastfeeding women during the consultations [22]. Enhancing human milk supply would be the primary anticipated effect of using fenugreek as a galactogogue. The panelists were of the opinion of informing the breastfeeding women recommended to use fenugreek of its antioxidant, estrogenic, and immunomodulatory properties $[35,43]$. Chemoprotective effects against breast cancer and antidepressant effects of fenugreek might also be communicated to and discussed with breastfeeding women $[27,35,43]$. Many breastfeeding women might be concerned with breast cancer and postpartum depression and could be interested in learning about these potential benefits of fenugreek. Breastfeeding women might also be informed of the antibacterial, antifungal, and antipyretic effects of fenugreek [42]. Fenugreek might also be beneficial in controlling appetite, promoting weight loss, alleviate ulcer, and decreasing cholesterol and triglycerides levels. Many breastfeeding women could have gained weight during pregnancy and might be interested in decreasing their weight. Fenugreek might offer some help toward this end.

The opinions of the panelists were divisive on the importance of communicating and discussing potential effects of fenugreek in inducing thirst, marble like urine and sweat. Similarly, the opinions of the panelists were divisive whether to communicate to and discuss with breastfeeding women potential benefits of fenugreek related to enhancing cognition, memory, and its antiparkinsonian effects [36, 43]. These potential harms and benefits might be or might not be discussed depending on the needs of each individual case.

In general, care should be taken when breastfeeding women are recommended treatments as many medications and herbal remedies are excreted into the human milk. Therefore, both breastfeeding women and their breastfed infants could be vulnerable. In all cases, potential benefits should be weighed against potential risks considering other available safe alternatives. Similar measures should be applied when fenugreek-based herbal remedies are intended to be recommended as galactogogues for breastfeeding women seeking recommendations to enhance their human milk supply.

The findings of this study could be interpreted considering a number of limitations. First, this was an observational consensual study. Observing healthcare provider's recommendations of fenugreek in daily clinical practice and why it was recommended for breastfeeding women could have shown other findings. Second, in this study, we did not classify potential harms and benefits into major harms and minor harms. However, this classification goes beyond the scope and objectives of this study. Third, we did not hierarchize the potential harms and benefits in order of importance. The hierarchy would have helped healthcare providers to prioritize the information to be communicated and discussed in case they did not have enough time to go over all potential benefits and harms. Fourth, judgmental and snowball sampling techniques were used to recruit panelists for this study. These nonprobability sampling techniques are viewed as biased in conservative views. However, these techniques are commonly used for this type of studies as probability sampling techniques are not practically feasible. Finally, the number of panelists who participated in each panel was relatively small. However, there is no consensus on the number of panelists required for a Delphi technique. The number of panelists used in this study was slightly larger than sizes used in previous studies seeking consensus on issues in healthcare.

\section{Conclusion}

Panelists in this study were of the opinion that potential harms and benefits of recommending the use of fenugreek 
as herbal galactogogue for breastfeeding women seeking recommendations to increase their human milk supply need to be discussed during the clinical consultations. This could be important in promoting congruence in daily healthcare delivery, improving patient's experience with therapy, coping with side effects of the therapy, and enhancing patient reported quality of life. In this study, consensus was achieved on a core list of potential harms and benefits of using fenugreek as herbal galactogogue in breastfeeding women seeking recommendations to enhance their human milk supply that need to be communicated to and discussed with breastfeeding women during the consultations in which fenugreek-based herbal remedies are to be recommended. This consensual list might be consulted as guidance by healthcare providers who are often consulted by breastfeeding women seeking recommendations to enhance their human milk supply. Further randomized clinical trials are still required to establish evidence-based benefits and harms of fenugreek in breastfeeding women. More observational studies are needed to assess what is being communicated and discussed in daily consultations when herbal remedies are recommended.

\section{Conflicts of Interest}

The authors declare that there are no conflicts of interest.

\section{Supplementary Materials}

Supplementary Table S1 provides the sociodemographic and practice details of the key contacts who were interviewed in this study $(n=15)$. Supplementary Table S2 provides details of the plants cited by the key contacts who were interviewed in this study $(n=15)$. (Supplementary Materials)

\section{References}

[1] E. A. Brownell, J. I. Hagadorn, M. M. Lussier et al., "Optimal periods of exclusive breastfeeding associated with any breastfeeding duration through one year," Journal of Pediatrics, vol. 166, no. 3, pp. 566-570, 2015.

[2] C. G. Victora, R. Bahl, A. J. D. Barros et al., "Breastfeeding in the 21st century: Epidemiology, mechanisms, and lifelong effect," The Lancet, vol. 387, no. 10017, pp. 475-490, 2016.

[3] A. Brown, "Breastfeeding as a public health responsibility: a review of the evidence," Journal of Human Nutrition and Dietetics, vol. 30, no. 6, pp. 759-770, 2017.

[4] F. McAndrew, Infant feeding survey 2010. Leeds: Health and Social Care Information Centre, 2012.

[5] L. E. Grzeskowiak, J. A. Dalton, and A. L. Fielder, "Factors associated with domperidone use as a galactogogue at an australian tertiary teaching hospital," Journal of Human Lactation, vol. 31, no. 2, pp. 249-253, 2015.

[6] A. M. Stuebe, B. J. Horton, E. Chetwynd, S. Watkins, K. Grewen, and S. Meltzer-Brody, "Prevalence and risk factors for early, undesired weaning attributed to lactation dysfunction," Journal of Women's Health, vol. 23, no. 5, pp. 404-412, 2014.

[7] B. Haase, S. N. Taylor, J. Mauldin, T. S. Johnson, and C. L. Wagner, "Domperidone for Treatment of Low Milk Supply in Breast Pump-Dependent Mothers of Hospitalized Premature
Infants: A Clinical Protocol," Journal of Human Lactation, vol. 32, no. 2, pp. 373-381, 2015.

[8] L. E. Grzeskowiak, S. W. Lim, A. E. Thomas, U. Ritchie, and A. L. Gordon, "Audit of domperidone use as a galactogogue at an Australian tertiary teaching hospital," Journal of Human Lactation, vol. 29, no. 1, pp. 32-37, 2013.

[9] A. Osadchy, M. E. Moretti, and G. Koren, "Effect of domperidone on insufficient lactation in puerperal women: a systematic review and meta-analysis of randomized controlled trials," Obstetrics and Gynecology International, vol. 2012, Article ID 642893, 7 pages, 2012.

[10] L. E. Grzeskowiak and L. H. Amir, "Use of domperidone to increase breast milk supply: Further consideration of the benefit-risk ratio is required," Journal of Human Lactation, vol. 31, no. 2, pp. 315-316, 2015.

[11] L. Grzeskowiak, "Use of Domperidone to increase breast milk supply: Are women really dying to breastfeed?" Journal of Human Lactation, vol. 30, no. 4, pp. 498-499, 2014.

[12] S. A. Doggrell and J. C. Hancox, "Cardiac safety concerns for domperidone, an antiemetic and prokinetic, and galactogogue medicine," Expert Opinion on Drug Safety, vol. 13, no. 1, pp. 131138, 2014.

[13] S. C. Foong, M. L. Tan, L. A. Marasco, J. J. Ho, and W. C. Foong, "Oral galactagogues for increasing breast-milk production in mothers of non-hospitalised term infants," Cochrane Database of Systematic Reviews, vol. 4, 2015.

[14] C. Paul, M. Zénut, A. Dorut et al., "Use of domperidone as a galactagogue drug: a systematic review of the benefit-risk ratio," Journal of Human Lactation, vol. 31, no. 1, pp. 57-63, 2015.

[15] R. Shawahna and A. Taha, "Which potential harms and benefits of using ginger in the management of nausea and vomiting of pregnancy should be addressed? A consensual study among pregnant women and gynecologists," BMC Complementary and Alternative Medicine, vol. 17, no. 1, article no. 204, 2017.

[16] H. Liu, Y. Hua, H. Luo, Z. Shen, X. Tao, and X. Zhu, "An Herbal Galactagogue Mixture Increases Milk Production and Aquaporin Protein Expression in the Mammary Glands of Lactating Rats," Evidence-Based Complementary and Alternative Medicine, vol. 2015, Article ID 760585, 2015.

[17] S. Colaceci, A. Giusti, A. De Angelis et al., "Medications, "natural" Products, and Pharmacovigilance during Breastfeeding: A Mixed-Methods Study on Womens Opinions," Journal of Human Lactation, vol. 32, no. 2, pp. 324-332, 2015.

[18] M. R. Amer, G. C. Cipriano, J. V. Venci, and M. A. Gandhi, "Safety of popular herbal supplements in lactating women," Journal of Human Lactation, vol. 31, no. 3, pp. 348-353, 2015.

[19] L. Gori, E. Gallo, V. Mascherini, A. Mugelli, A. Vannacci, and F. Firenzuoli, "Can estragole in fennel seed decoctions really be considered a danger for human health? A fennel safety update," Evidence-Based Complementary and Alternative Medicine, vol. 2012, Article ID 860542, 10 pages, 2012.

[20] S. B. Badgujar, V. V. Patel, and A. H. Bandivdekar, "Foeniculum vulgare Mill: A review of its botany, phytochemistry, pharmacology, contemporary application, and toxicology," BioMed Research International, vol. 2014, Article ID 842674, 2014.

[21] M. Mortel and S. D. Mehta, "Systematic review of the efficacy of herbal galactogogues," Journal of Human Lactation, vol. 29, no. 2, pp. 154-162, 2013.

[22] T. M. Khan, D. B.-C. Wu, and A. V. Dolzhenko, "Effectiveness of fenugreek as a galactagogue: A network meta-analysis," Phytotherapy Research, 2017. 
[23] A. Albassam and A. Awad, "Community pharmacists' services for women during pregnancy and breast feeding in Kuwait: a cross-sectional study," BMJ Open, vol. 8, no. 1, p. e018980, 2018.

[24] M. P. Gabay, "Galactogogues: medications that induce lactation," Journal of Human Lactation, vol. 18, no. 3, pp. 274-279, 2002.

[25] K. Huggins, Fenugreek: One Remedy for Low Milk Production. Breastfeeding Online, 2017.

[26] H.-T. Chan, L.-T. So, S.-W. Li, C.-W. Siu, C.-P. Lau, and H.-F. Tse, "Effect of herbal consumption on time in therapeutic range of warfarin therapy in patients with atrial fibrillation," Journal of Cardiovascular Pharmacology, vol. 58, no. 1, pp. 87-90, 2011.

[27] K. El Bairi, M. Ouzir, N. Agnieszka, and L. Khalki, "Anticancer potential of Trigonella foenum graecum: Cellular and molecular targets," Biomedicine \& Pharmacotherapy, vol. 90, pp. 479491, 2017.

[28] C. K. Fæste, E. Namork, and H. Lindvik, "Allergenicity and antigenicity of fenugreek (Trigonella foenum-graecum) proteins in foods," The Journal of Allergy and Clinical Immunology, vol. 123, no. 1, pp. 187-194, 2009.

[29] K. Hamden, H. Keskes, S. Belhaj, K. Mnafgui, A. Feki, and N. Allouche, "Inhibitory potential of omega-3 fatty and fenugreek essential oil on key enzymes of carbohydrate-digestion and hypertension in diabetes rats," Lipids in Health and Disease, vol. 10, article no. 226, 2011.

[30] A. A. Izzo, G. di Carlo, F. Borrelli, and E. Ernst, "Cardiovascular pharmacotherapy and herbal medicines: the risk of drug interaction," International Journal of Cardiology, vol. 98, no. 1, pp. 1-14, 2005.

[31] N. A. Jaradat, R. Shawahna, A. M. Eid, R. Al-Ramahi, M. K. Asma, and A. N. Zaid, "Herbal remedies use by breast cancer patients in the West Bank of Palestine," Journal of Ethnopharmacology, vol. 178, pp. 1-8, 2016.

[32] A. D. Kaye, R. C. Clarke, R. Sabar et al., "Herbal medicines: Current trends in anesthesiology practice - A hospital survey," Journal of Clinical Anesthesia, vol. 12, no. 6, pp. 468-471, 2000.

[33] L. Khalki, S. B. M'hamed, M. Bennis, A. Chait, and Z. Sokar, "Evaluation of the developmental toxicity of the aqueous extract from Trigonella foenum-graecum (L.) in mice," Journal of Ethnopharmacology, vol. 131, no. 2, pp. 321-325, 2010.

[34] R. Mebazaa, B. Rega, and V. Camel, "Analysis of human male armpit sweat after fenugreek ingestion: Characterisation of odour active compounds by gas chromatography coupled to mass spectrometry and olfactometry," Food Chemistry, vol. 128, no. 1, pp. 227-235, 2011.

[35] K. C. Nagulapalli Venkata, A. Swaroop, D. Bagchi, and A. Bishayee, "A small plant with big benefits: Fenugreek (Trigonella foenum-graecum Linn.) for disease prevention and health promotion," Molecular Nutrition \& Food Research, vol. 61, no. 6, Article ID 1600950, 2017.

[36] J. Nathan, S. Panjwani, V. Mohan, V. Joshi, and P. A. Thakurdesai, "Efficacy and safety of standardized extract of Trigonella foenum-graecum 1 seeds as an adjuvant to L-dopa in the management of patients with Parkinson's disease," Phytotherapy Research, vol. 28, no. 2, pp. 172-178, 2014.

[37] C. Necyk and L. Zubach-Cassano, "Natural Health Products and Diabetes: A Practical Review," Canadian Journal of Diabetes, vol. 41, no. 6, pp. 642-647, 2017.

[38] M. Ouzir, K. El Bairi, and S. Amzazi, “Toxicological properties of fenugreek (Trigonella foenum graecum)," Food and Chemical Toxicology, vol. 96, pp. 145-154, 2016.
[39] R. Samavati, E. Ducza, J. Hajagos-Tóth, and R. Gaspar, "Herbal laxatives and antiemetics in pregnancy," Reproductive Toxicology, vol. 72, pp. 153-158, 2017.

[40] C. R. Sirtori, C. Galli, J. W. Anderson, E. Sirtori, and A. Arnoldi, "Functional foods for dyslipidaemia and cardiovascular risk prevention," Nutrition Research Reviews, vol. 22, no. 2, pp. 244261, 2009.

[41] I. M. Taj Eldin, M. M. Abdalmutalab, and H. E. Bikir, "An in vitro anticoagulant effect of Fenugreek (Trigonella foenumgraecum) in blood samples of normal Sudanese individuals," Sudanese Journal of Paediatrics, vol. 13, no. 2, pp. 52-56, 2013.

[42] U. C. S. Yadav and N. Z. Baquer, "Pharmacological effects of Trigonella foenum-graecum L. in health and disease," Pharmaceutical Biology, vol. 52, no. 2, pp. 243-254, 2014.

[43] S. Zameer, A. K. Najmi, D. Vohora, and M. Akhtar, “ A review on therapeutic potentials of ," Nutritional Neuroscience, pp. 1-7, 2017.

[44] A. N. Bazzano, R. Hofer, S. Thibeau, V. Gillispie, M. Jacobs, and K. P. Theall, "A review of herbal and pharmaceutical galactagogues for breast-feeding," The Ochsner Journal, vol. 16, no. 4, pp. 511-524, 2016.

[45] M. J. Stanger, L. A. Thompson, A. J. Young, and H. R. Lieberman, "Anticoagulant activity of select dietary supplements," Nutrition Reviews, vol. 70, no. 2, pp. 107-117, 2012.

[46] Z. Chen et al., "Effects of Saponin from Trigonella FoenumGraecum Seeds on Dyslipidemia," Iranian Journal of Medical Sciences, vol. 42, no. 6, pp. 577-585, 2017.

[47] R. Shawahna, "Which information on women's issues in epilepsy does a community pharmacist need to know? A Delphi consensus study," Epilepsy \& Behavior, vol. 77, pp. 79-89, 2017.

[48] R. Shawahna, A. Haddad, B. Khawaja, R. Raie, S. Zaneen, and T. Edais, "Medication dispensing errors in Palestinian community pharmacy practice: a formal consensus using the Delphi technique," International Journal of Clinical Pharmacy, vol. 38, no. 5, pp. 1112-1123, 2016.

[49] R. Shawahna, D. Masri, R. Al-Gharabeh, R. Deek, L. AlThayba, and M. Halaweh, "Medication administration errors from a nursing viewpoint: A formal consensus of definition and scenarios using a Delphi technique," Journal of Clinical Nursing, vol. 25, no. 3-4, pp. 412-423, 2016.

[50] S. Njuangang, C. Liyanage, and A. Akintoye, "Application of the Delphi technique in healthcare maintenance," International Journal of Health Care Quality Assurance, vol. 30, no. 8, pp. 737754, 2017.

[51] A. Page, K. Potter, R. Clifford, A. McLachlan, and C. EthertonBeer, "Prescribing for Australians living with dementia: Study protocol using the Delphi technique," BMJ Open, vol. 5, no. 8, Article ID e008048, 2015.

[52] A. N. Bazzano, L. Littrell, A. Brandt, S. Thibeau, K. Thriemer, and K. P. Theall, "Health provider experiences with galactagogues to support breastfeeding: A cross-sectional survey," Journal of Multidisciplinary Healthcare, vol. 9, pp. 623-630, 2016.

[53] R. Shawahna and N. A. Jaradat, "Ethnopharmacological survey of medicinal plants used by patients with psoriasis in the West Bank of Palestine," BMC Complementary and Alternative Medicine, vol. 17, no. 1, article no. 4, 2017.

[54] S. M. Dunlay and J. J. Strand, "How to discuss goals of care with patients," Trends in Cardiovascular Medicine, vol. 26, no. 1, pp. 36-43, 2016.

[55] L. E. Grzeskowiak, M. Hill, and D. S. Kennedy, "Phone calls to an Australian pregnancy and lactation counselling service regarding use of galactagogues during lactation - the MotherSafe 
experience," Australian and New Zealand Journal of Obstetrics and Gynaecology.

[56] D. Tiran, "Ginger to reduce nausea and vomiting during pregnancy: evidence of effectiveness is not the same as proof of safety," Complementary Therapies in Clinical Practice, vol. 18, no. 1, pp. 22-25, 2012. 


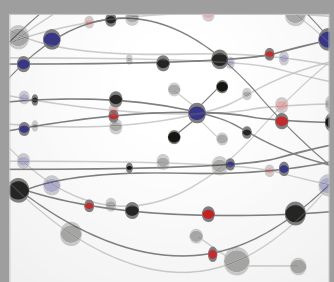

The Scientific World Journal
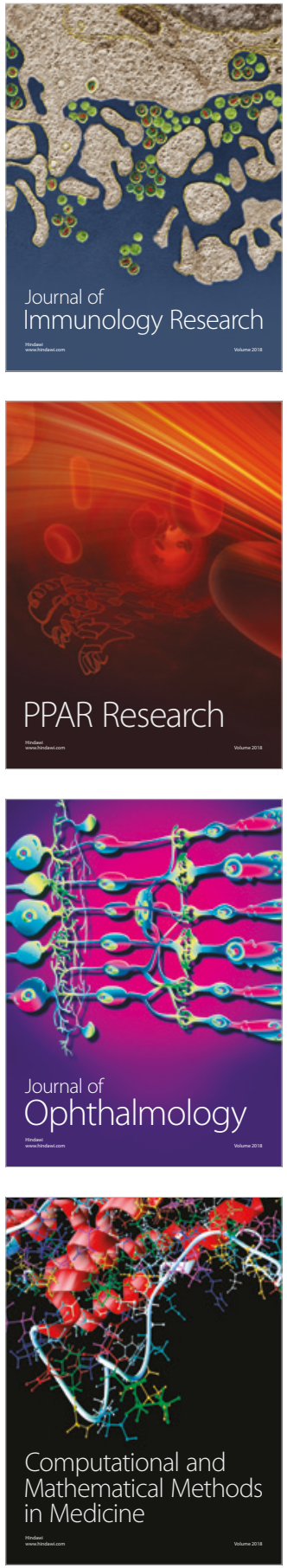

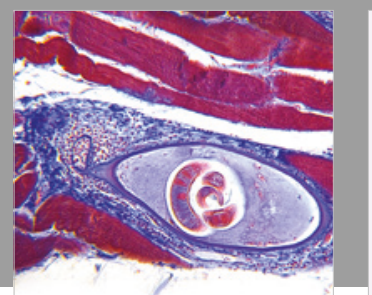

Gastroenterology Research and Practice

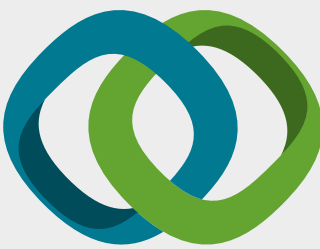

\section{Hindawi}

Submit your manuscripts at

www.hindawi.com
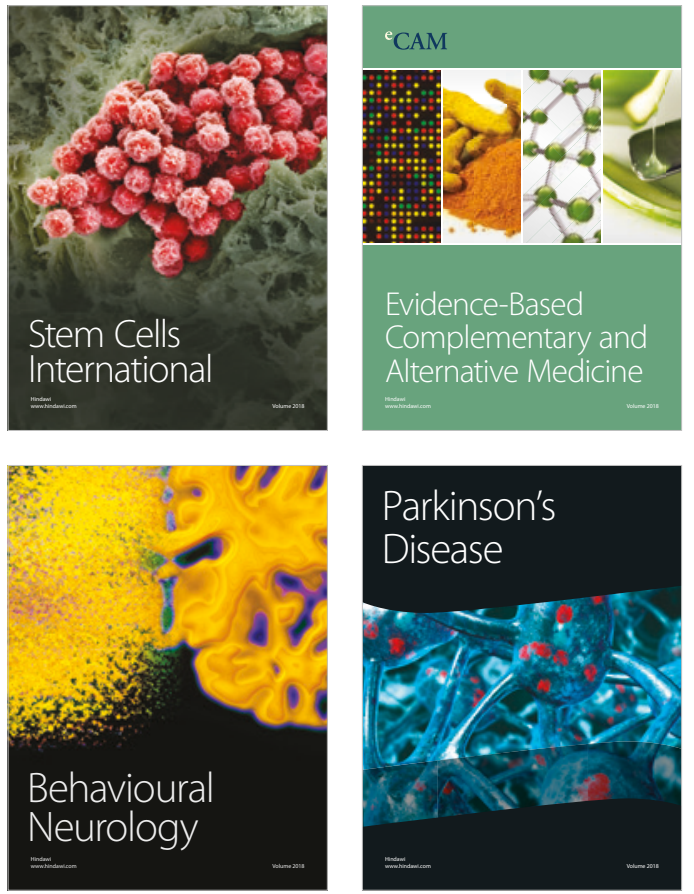

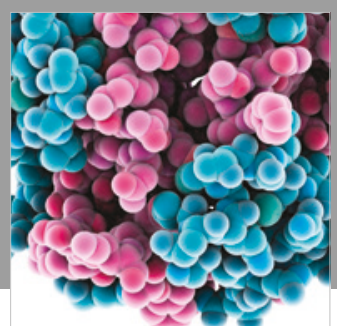

ournal of

Diabetes Research

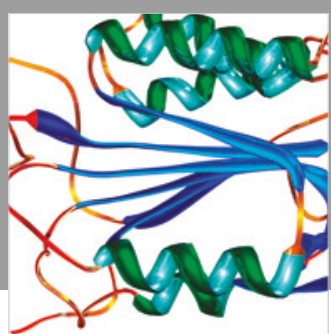

Disease Markers
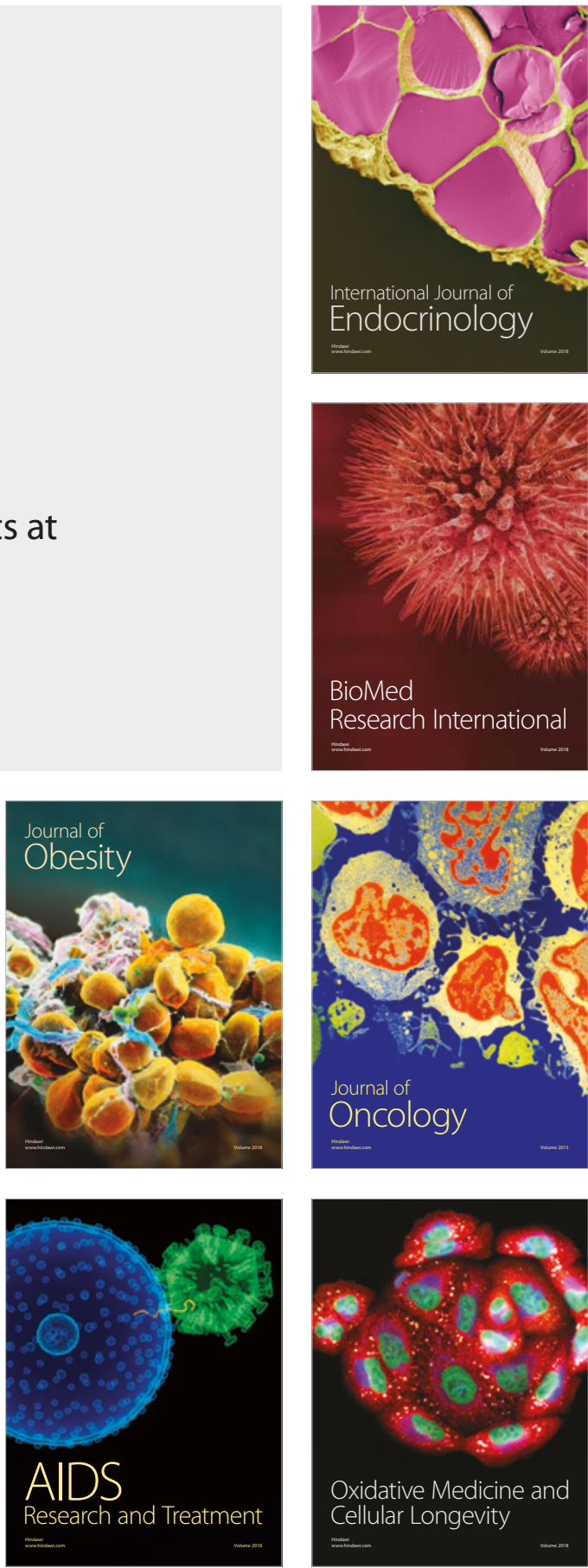